\title{
Learning From Nature: Emulating Macromolecular Crowding To Drive Extracellular Matrix Enhancement For The Creation Of Connective Tissue in vitro
}

\author{
Yanxian Peng and Michael Raghunath \\ National University of Singapore \\ Singapore
}

\section{Introduction}

Tissue engineering, a relatively new field, combines a multitude of separate disciplines: clinical medicine, cell biology, engineering, materials science, biochemistry and also biophysics. With such a diverse pool of research backgrounds, tissue engineers seek a common goal: to replace or repair part of or whole tissues and organs, with functional manmade tissue constructs.

To date, there have been attempts to tissue-engineer bone, cartilage, liver and various other tissues and organs. The more successful tissue engineered constructs have been simpler tissues such as artificial skin, bladder and recently, tissue engineered airway (Macchiarini et. al. 2008). As tissue complexity increases, achieving fully functional tissue constructs becomes exponentially more difficult.

Conventional tissue-engineering strategies can be broadly classified into cell-free and cellbased therapies. Both strategies typically utilize scaffolds, with or without cells, and typically provide biologically active molecules to enhance integration into the body. Scaffolds serve many purposes: mechanical support, cell migration and attachment, cell retention and delivery at the site of repair, and even sequester biochemical factors and/or genes, while allowing for diffusion of nutrients from the host tissue into the construct. Essentially, an ideal scaffold will act as a man-made emulation of the natural extracellular matrix found (ubiquitously) in the body.

Scaffold materials come in many different forms, from synthetic to nature-derived materials, each with a set of properties defining their intrinsic advantages and limitations for application. Polymer scaffold materials have been a mainstay of tissue-engineered constructs, however there has been an increasing awareness of the advantages of biomimicry or nature-derived scaffolds often fabricated from native and denatured "collagen", fibrin, chitosan and glycosaminoglycans. These scaffolds are more biocompatible but remain simplified copies of the complex extracellular milieu in living tissue. 
The extracellular matrix is a complex supramolecular assembly of a variety of glycoproteins and proteoglycans. Extracellular matrix provides tissue integrity, acts as a native scaffold for cell attachment and interaction and also serves as a reservoir for growth factors. For most connective tissues, collagen makes up the bulk of the extracellular matrix. Collagen functions in the extracellular matrix as a structural protein as well as a binding partner for ligands that store growth factors. The term collagen encompasses a family of glycoproteins characterised by a particular amino acid signature and triplehelicity. The most abundant collagens are type I, found in skin, tendon and capsules, type IV, found in all basement membranes, and type VII, which is specific for the basement membrane of skin, oral mucosa and cornea. Depending on their tissue location and function, collagen assemblies in the extracellular matrix are often heterotypic. For example, collagen fibers in the dermis represent an alloy of types I, III and V, while cartilage matrix contains types II, IX, and XI (Kielty \& Grant, 2002). Depending on the wear and tear of a given tissue, extracellular matrix continuously undergoes remodeling and replacement.

Tissue engineers have long underestimated the capacity of cells to manufacture their own extracellular matrix. We show in the following text, ways to control and augment this cellular capacity to create a well-developed extracellular matrix that can provide cellular cohesion and tissue strength. This cellular ability is an important quality required for downstream applications of tissue engineering. Enhancing the deposition of collagen in tissue-engineered constructs will aid in the maturity of the tissue construct in vitro prior to clinical application as well as provide a functional model for the study of fibrogenic processes for therapeutic purposes.

\section{Application of Macromolecular Crowding (MMC) in Enhancing Matrix Deposition}

\subsection{In vitro collagen matrix formation (deposition) is tardy}

The typical signature of a collagen molecule is its quaternary structure - the triplehelix. It is a trimer of $\alpha$-chains that is assembled intracellulary (Kielty \& Grant, 2002) and secreted by fibrogenic cells into the extracellular space as soluble procollagen. Taking procollagen type I as the most abundant representative, one can envision the polypeptide as a dumbbellshaped molecule with a central triple helical region of rod-like stiffness and globular propeptide at either end. Once in the extracellular space, both propeptides of procollagen are cleaved off by procollagen $\mathrm{N}$-proteinase and procollagen C-proteinase respectively. The resulting collagen molecules will then self-assemble in vivo at $\mathrm{pH}$ 7-7.4 into quarterstaggered arrays, thus initiating fibrillogenesis (Kielty \& Grant, 2002).

When adequately provided with ascorbic acid (AcA), fibrogenic cells secrete substantial amounts of procollagen (Murad et. al., 1981). However, the enzymatic conversion of procollagen to collagen is extremely tardy under contemporary aqueous cell culture conditions, a fact that is not widely known. Consequently, only small amounts of an insoluble collagen matrix are formed, while most of the (water soluble) procollagen is lost during culture medium change and in bioreactor outflows (Lareu et. al., 2007a, b). 


\subsection{In vitro collagen biosynthesis is enhanced by MMC - effects of negatively charged crowders}

We have found that the addition of negatively-charged macromolecules for two days during the culture of fibroblasts reduces procollagen content in the culture medium and enhances the amount of (processed) collagen molecules deposited at the cell layer.

Fibroblasts were first grown in culture medium with or without AcA and macromolecules. The procollagen in the culture medium and the collagen found in the adherent cell layer were isolated by pepsin and quantified by sodium dodecyl sulfate polyacrylamide gel electrophoresis and densitometry as described by Lareu et. al. 2007a, b. Fibroblasts that were not treated with AcA secreted little procollagen into the culture medium and therefore showed no appreciable deposition of collagen deposited into the matrix. ${ }^{1}$ Under AcA supplementation the amount of procollagen secreted into the culture medium was substantially increased. However, there was little insoluble collagen at the adherent cell layer. However, with the addition of $100 \mu \mathrm{g} / \mathrm{ml}$ of $500 \mathrm{kDa}$ dextran sulfate (DxS) for two days, the presence of procollagen in the medium was much reduced, while the amount of collagen associated with the cell layer was dramatically increased (Fig. 1.).

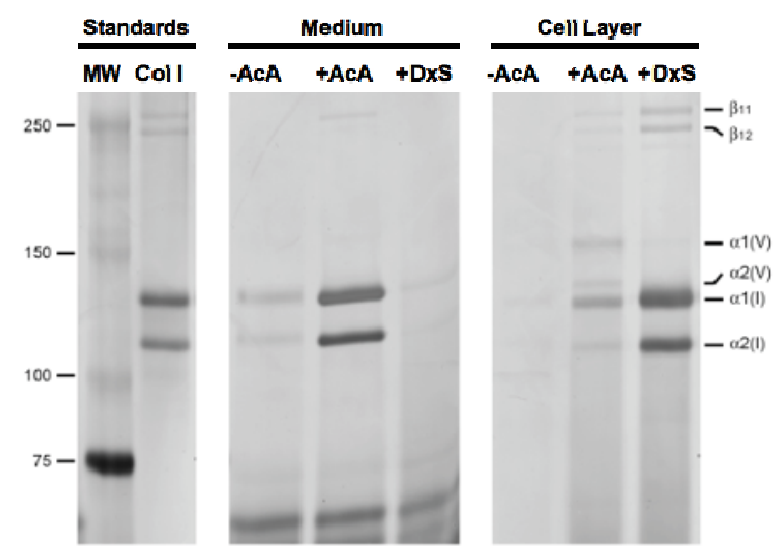

Fig. 1. Addition of $100 \mu \mathrm{g} / \mathrm{ml}$ of $500 \mathrm{kDa}$ DxS resulted in the reduction of procollagen in the culture medium and the increase of insoluble collagen at the fibroblast cell layer. Note the predominance of collagen $\mathrm{V}$ in the non-crowded cell layer. Reprinted from Lareu et. al., 2007a, with permission from the publisher, Mary Ann Liebert

DxS concentrations ranging from $10 \mu \mathrm{g} / \mathrm{ml}$ to $500 \mu \mathrm{g} / \mathrm{ml}$ were applied to fibroblast cultures. It was observed that collagen bands from cell layer samples became more intense with an

\footnotetext{
1 AcA is an essential cofactor for prolyl-4-hydroxylase, an enzyme that catalyses a crucial posttranslational modification to the $\alpha$-chains resulting in thermal stability (at body temperature) of the folded procollagen triplehelix in the endoplasmic reticulum. In the absence of AcA, prolyl residues of individual $\alpha$-chains are not sufficiently hydroxylated of form a thermostable triplehelix that can be secreted. (Murad et. al., 1981). Therefore, the omission of ascorbate causes the equivalent of scurvy in culture. Similar to the clinical condition, this can be remedied easily.
} 
increasing concentration of DxS up to $100 \mu \mathrm{g} / \mathrm{ml}$ (Fig. 2.). At $500 \mu \mathrm{g} / \mathrm{ml}$, the intensity of collagen bands decreased sharply and, hence, we concluded that the optimum concentration for crowding with DxS was $50-100 \mu \mathrm{g} / \mathrm{ml}$.
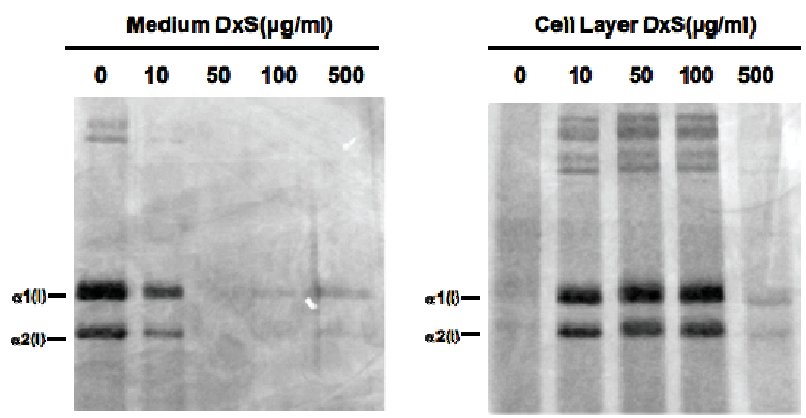

Fig. 2. Collagen deposition was enhanced with increasing concentration of DxS up to $100 \mu \mathrm{g} / \mathrm{ml}$. At $500 \mu \mathrm{g} / \mathrm{ml}$, collagen deposition decreased sharply. The optimum concentration of DxS was determined to be $50-100 \mu \mathrm{g} / \mathrm{ml}$. Reprinted from Lareu et. al., 2007a, with permission from the publisher, Mary Ann Liebert

We also found a marked increase of collagen deposition with 200kDa polysodium-4-styrene sulfonate (PSS), a negatively-charged macromolecule, at a final concentration of $100 \mu \mathrm{g} / \mathrm{ml}$ culture medium in fibroblast cultures for two days. Collagen in the adherent cell layer was again isolated by pepsin digestion and analyzed by sodium dodecyl sulfate-polyacrylamide gel electrophoresis. Densitometric analysis of collagen $\alpha 1(\mathrm{I})$ and $\alpha 2(\mathrm{I})$ bands (Fig. 3A) revealed that the addition of DxS increased collagen deposition by $>20$-fold, and the addition of PSS increased collagen deposition by $>35$-fold (Fig. 3B). We have also tested the efficacy of other macromolecules in increasing collagen deposition.. These macromolecules, $50 \mathrm{mg} / \mathrm{ml}$ of $400 \mathrm{kDa}$ Ficoll (Fc 400), 50mg/ml of 70kda Ficoll (Fc 70) and 100 $\mu \mathrm{g} / \mathrm{ml}$ of $10 \mathrm{kDa}$ DxS (DxS 10), did not induce a significant increase in collagen deposition at the cell layer in the time window of 2 days (Fig. 3A.)
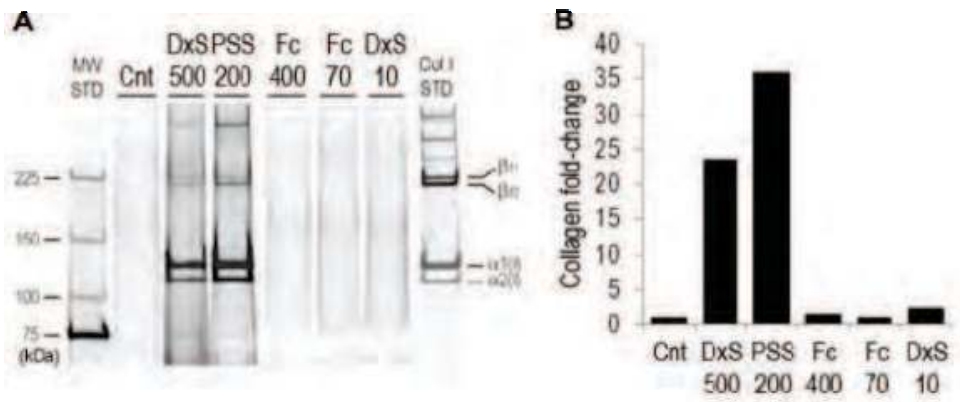

Fig. 3. $100 \mu \mathrm{g} / \mathrm{ml}$ of DxS increased collagen deposition by $>20$-fold. $100 \mu \mathrm{g} / \mathrm{ml}$ of PSS was also shown to induce an increase in collagen deposition by $>35$-fold. Reprinted from Lareu et. al., 2007b, with permission from the publisher, Elsevier 


\subsection{MMC via negatively charged macromolecules results in a granular collagen deposition}

Immunocytochemical analyses of fibroblast cultures treated with DxS or PSS corroborated the SDS-PAGE results. There were significantly stronger signals of collagen immunostaining in these fibroblast cultures compared to the uncrowded control (Fig. 4A.). With regard to the deposition pattern, we noted that the collagen aggregated in a granular form, co-localized with fibronectin staining, whereas the minimal collagen deposited in the uncrowded control was reflected by weakly stained wispy strands.

Transmission electron microscopy revealed that these aggregates produced under DxS crowding contained cross-striated collagen nanofibers surrounded by a three dimensional arrangement of other matrix components (Fig 4B.) We therefore address these aggregates as "microgels".

A
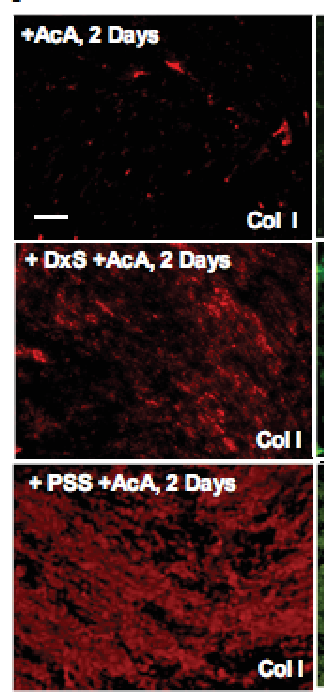

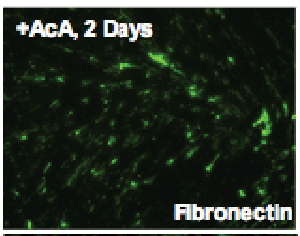

+ Dxs + AcA, 2 Days
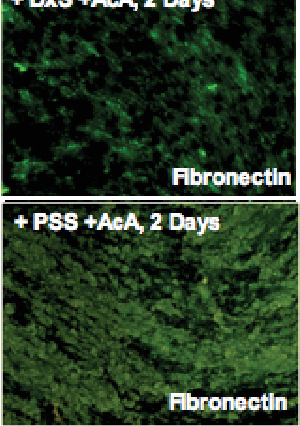

B

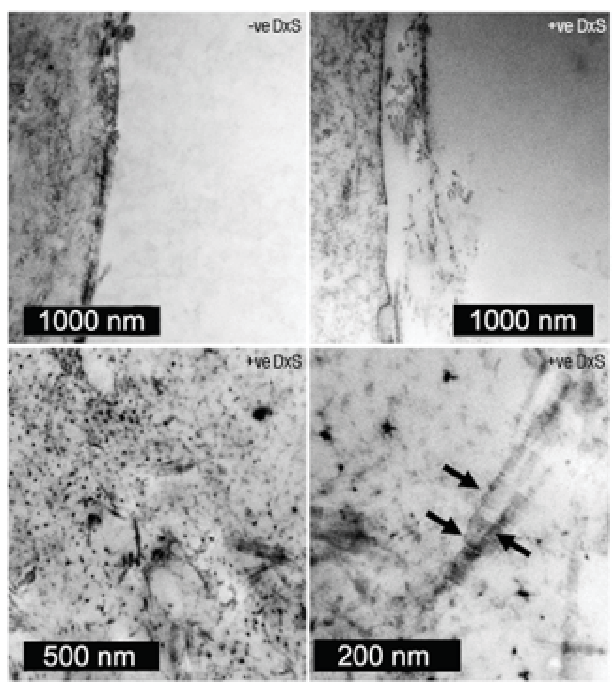

Fig. 4. Morphological analysis of fibroblast cultures in the absence and presence of DxS. (A) Double immunfluorescence staining for collagen and fibronectin reveals granular morphology for DxS-crowded and PSS-crowded fibroblast cell layers. The uncrowded control showed wispy strands of collagen and fibronectin. All magnifications at 10X, scale bar100 $\mu \mathrm{m}$; (B) Transmission electron micrograph of the pericellular matrix in the absence and presence of DxS. Arrows indicate typical collagen fibrillar formation in the presence of DxS. In the presence of DxS, fibroblast cell layer samples showed cross-striated collagen nanofibers, which were absent in non-crowded fibroblast cultures. Fig. 4B was reprinted from Lareu et. al., 2007b, with permission from the publisher, Elsevier

\subsection{In vitro collagen biosynthesis is enhanced by MMC - effects of neutral crowders}

Although we initially found that Fc 400 and Fc 70 as monocrowders were unable to enhance collagen production significantly in two days, we discovered that a combination of Fc 400 and Fc 70 added as a cocktail to the fibroblast cultures increased collagen deposition $>10$ - 
fold in six days compared to non-crowded controls (Fig. 5A and 5B). Fc 400 and Fc 70 were used at concentrations of $25 \mathrm{mg} / \mathrm{ml}$ and $37.5 \mathrm{mg} / \mathrm{ml}$, respectively.

However, we noted with interest that under this cocktail, collagen matrices started out in a granular pattern (not shown) but transformed into a reticular pattern reminiscent of the pattern seen with fibronectin staining in the uncrowded controls. This observation is in keeping with the notion that fibronectin serves as a primordial template for collagen deposition (Sottile et. al., 2007). Staining of collagen in Fc-supplemented fibroblast cultures was much stronger in comparison to non-crowded controls. In accordance to earlier observations (see section 2.3), collagen deposits induced by DxS and PSS supplementation showed a coarse granular appearance also after six days (Fig. 5C).

A
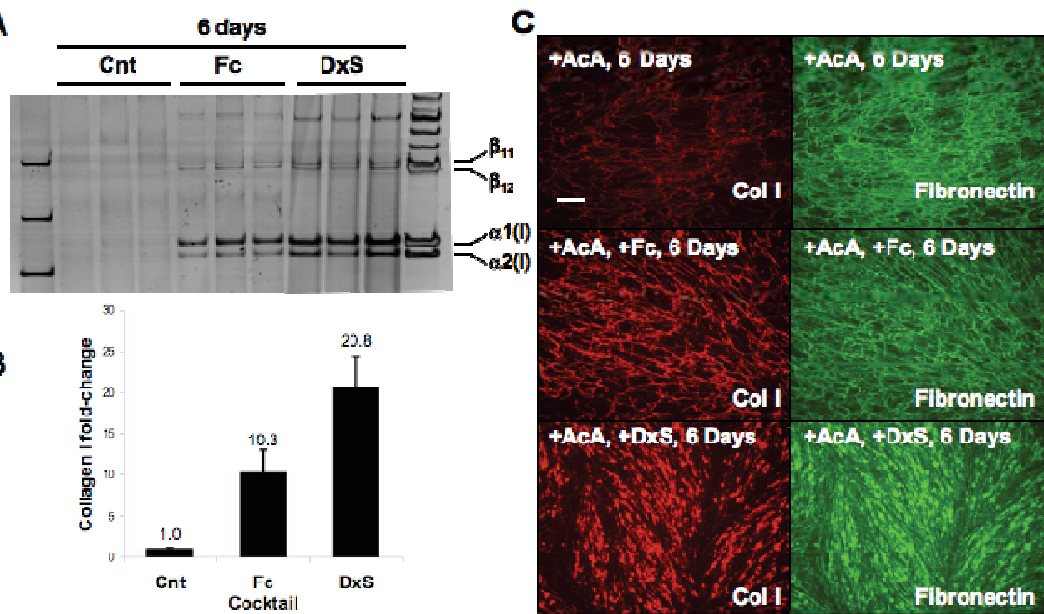

Fig. 5. Application of a mixture of neutral macromolecules Fc 400 and Fc 70 to fibroblast culture medium is also able to increase collagen deposition over the course of 6 days, resulting in a reticular network of collagen deposition rather than the granular collagen deposition observed with negatively charged crowders

\subsection{How MMC works - enhancing key enzymatic activities in collagen matrix formation} MMC is a biophysical principle in which inert macromolecules in solution occupy a significant volume of the medium and hence impact on biochemical reactions both in vivo and in vitro (Minton, 2001). This principle is highly conserved throughout evolution for the interior of cells and the extracellulular environment in multicellular organisms (Zimmerman \& Minton, 1993). The density or crowdedness of macromolecules is best determined first by approximation as solute content. To date, the concentrations of macromolecules in the interior of cells have been determined in a range from 50 to $400 \mathrm{mg} / \mathrm{ml}$ (Minton, 2001). This concentration refers to the cumulative concentration of all species of macromolecules present, rather than the concentration of any single macromolecule. The presence of these macromolecules means that they must significantly occupy space in the given medium, and biophysicists refer to such medium as "crowded" or "confining"(Minton, 2001).

In order to illustrate the principle of MMC, picture a simplified scenario where macromolecules (blue) are confined in a given amount of space (box) (Fig. 6.). When a 
reactant molecule (red) of a similar size is introduced to such a system, the presence of these macromolecules limit the space available (white area) to the reactant molecule. It is obvious that the limitation is due to the fact that no two molecules can overlap in the given space. Additionally, the limitation is also due to the dead space (black area) in between macromolecules that the reactant molecule is unable to pass through. Hence the excluded volume is the sum of the occupied space and the dead (or unavailable) space. In such a scenario, there are two consequences on the reactant molecule. Firstly, the reduction in volume will increase the effective concentration of the reactant molecule, and hence the thermodynamic activity of the reactant. Secondly, the diffusion rate and correspondingly the rate of encounter of a reactant molecule with another is also reduced (Chebotareva et. al., 2004). The first consequence will manifest in reactions where the stability of the transition state is limiting, resulting in increased reaction rates. On the other hand, the second consequence will manifest in reactions where the encounter rates are limiting, resulting in decreased reaction rates (Ellis, 2001).

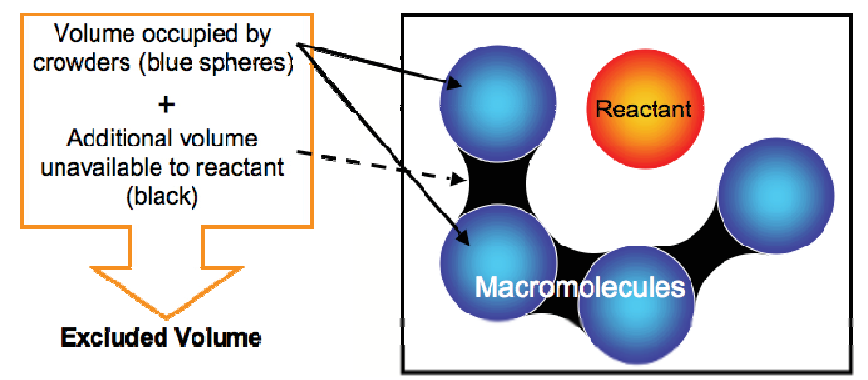

Fig. 6. Macromolecules (blue) limit the space available (white) for the reactant molecule (red) by occupying space and creating dead space (black) in between macromolecules. Both crowder volume and dead space volume contribute to the excluded volume available for the reactant molecule

In addition to the molecular size and shape attributes of a macromolecule, the hydration shells around such crowders also contribute to the total volume of exclusion as they are in an aqueous solvent. In addition, the surface charge on many of the ionic crowders, imparts an electrostatic contribution to this steric exclusion, as a result of like-charge repulsion, thus amplifying the steric exclusion effects.

Finally, the most effective increase in thermodynamic activities is exerted by like-sized molecules on each other (Ellis, 2001). If the reactant molecule is much larger or much smaller than the macromolecule, the effect is less significant.

In contrast to living systems, typical in vitro methods involving all kinds of settings (tube, cuvette, ELISA plate, cell culture dish) are done in aqueous solutions with concentrations of macromolecules ranging from 1 to $10 \mathrm{mg} / \mathrm{ml}$ (Ellis, 2001). Fibroblasts and other cells are typically cultured on plain or thinly coated tissue culture plastic, bathed in large volumes of non-crowded aqueous medium. This situation is far from physiological. In the body, extracellular space comprises of dense arrays of extracellular matrix macromolecules. Even blood plasma shows solute concentrations of around $80 \mathrm{mg} / \mathrm{ml}$ (Ellis, 2001). In contrast, typical cell cultures employ supplements of 5\%-20\% fetal bovine serum resulting in solute 
concentration of $4-16 \mathrm{mg} / \mathrm{ml}$. Obviously, such culture conditions - the current norm - do not provide a crowded environment.

In 1986, Bateman et. al. reported that when polyethylene glycol (PEG), dextran T-40 or polyvinylpyrrolidone were added to fibroblast cultures, no collagen or procollagen was found in the medium but instead was associated with the cell layer (Bateman et.al., 1986). In 1994, Hojima et. al. found that either the presence of $500 \mathrm{kDa}$ dextran sulfate or PEG increased the respective activities of purified procollagen C-proteinase and purified procollagen N-proteinase in vitro in a cell-free system (Hojima et.al., 1994). However, there was no follow-up on these publications until 2007, when we set out to use this system for translational purposes.

Under our control, the addition of inert macromolecules was able to dramatically enhance collagen deposition (Lareu et. al. 2007a,b). The macromolecules employed exerted a crowding effect on the pathways involved in collagen deposition. We have identified at least two enzymatic reactions that are boosted under MMC.

A

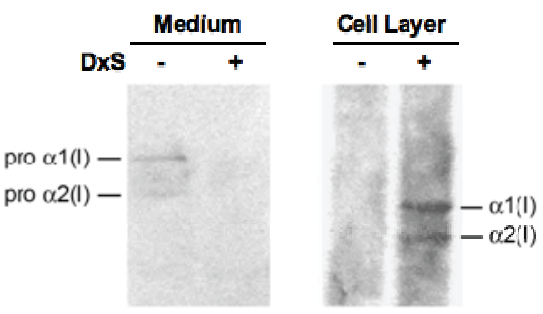

B

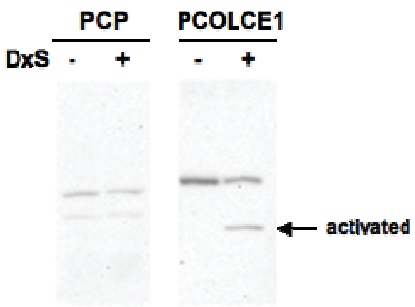

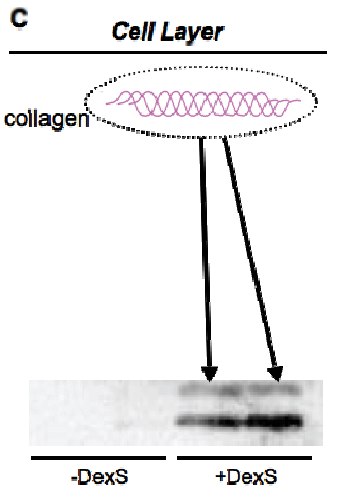

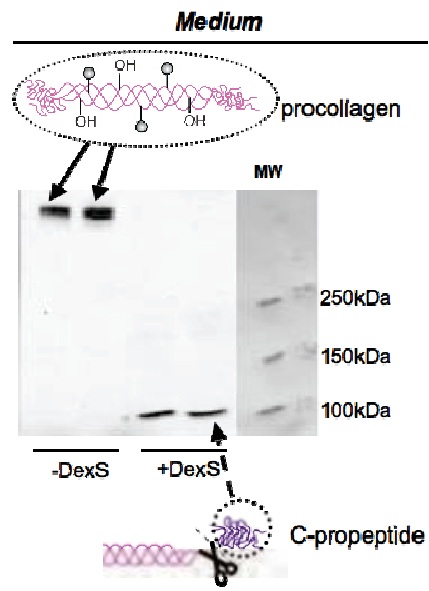

Fig. 7. Proteolytic cleavage of procollagen is enhanced by MMC. (A) Immunoblot for the central portion of collagen showing increased collagen staining from the cell layer fraction under MMC. In the absence of $\mathrm{MMC}$, procollagen remained unprocessed and in the medium fraction. (B) Immunoblot for PCP showed little significant change in quantity of PCP but immunoblot for PCOLCE1, an enhancer of PCP, showed increased activation. (C) Immunoblot for the C-propeptide showed the presence of higher molecular weight 
procollagen in the medium of uncrowded controls, and the presence of lower molecular weight C-propeptide in the medium of crowded fibroblast cultures. Fig. 7A was reprinted from Lareu et. al., 2007a, with permission from the publisher, Mary Ann Liebert. Fig. 7B was reprinted from Lareu et. al., 2007b, with permission from the publisher, Elsevier

Firstly, we observed an increase in procollagen conversion in the presence of DxS through Western blot analysis (Fig. 7). An antibody that recognizes only the central portion of the collagen molecule was used. Control cultures contained only procollagen in the medium, with little collagen detected in the cell layer. In the presence of DxS, procollagen in the medium was reduced. On the other hand, procollagen-processed collagen was present in the cell layer, indicating increased procollagen conversion into collagen and subsequent deposition at the cell layer (Fig. 7a). Western blot analysis of procollagen C-proteinase (PCP), the enzyme required for procollagen cleavage into collagen, revealed no significant changes in its amount (Fig. 7B) Therefore, we infer that its activity must have changed, which can be confirmed using antibodies against the C-propeptide (Fig 7C). Interestingly, we noted a proteolytic fragmentation under crowding with PCOLCE1, the allosteric enhancer of PCP-procollagen-specific activity (Fig. 7B). Only in the presence of DxS, did we detect a 36kDa active form of PCOLCE along with a C-terminal fragment, which was shown to inhibit matrix metalloproteinase 2 (MMP2) (Mott et.al., 2000).

A second identified enzymatic reaction is the lysyl oxidase-catalyzed reaction which produces large molecular weight dimers, $\beta_{11}$ and $\beta_{12}$, resulting in covalent crosslinking of collagen assemblies. Under crowded conditions, we determined via densitometry an increase of the ratio of $\beta$ bands versus $\alpha$ bands, suggesting an increased lysyl oxidasemediated crosslinking of the deposited collagen. (Chen et. al., 2009)

Hence, we hypothesize that MMC influences collagen production and turnover, and thus extracellular matrix production and retention, by at least three ways (Fig. 8). Under the influence of $\mathrm{MMC}$, there is increased proteolytic conversion of a procollagen C-proteinase allosteric enhancer, PCOLCE1, into its $36 \mathrm{kDa}$ active form and a $16.5 \mathrm{kDa}$ fragment that acts as a MMP2 inhibitor. The former will then enhance PCP enzymatic activity, resulting in increased procollagen cleavage in the extracellular space, thereby producing water-insoluble collagen molecules. These collagen molecules will then self-assemble in a quarter-staggered array at the cell layer. Moreover, the binding of PCOLCE1 to procollagen C-proteinase would be enhanced by MMC. MMC also enhances lysyl oxidase activity, which catalyzes the $\beta$ crosslinks between the collagen molecules, and so stabilizing these collagen molecules. The other by-product of PCOLCE1 conversion results in a MMP2 inhibitor, which aids in reducing the collagen turnover and thus maintaining the collagen matrix. Finally, crowding might also drive the supramolecular assembly of collagen, as we have observed in gel forming assays (unpublished). 


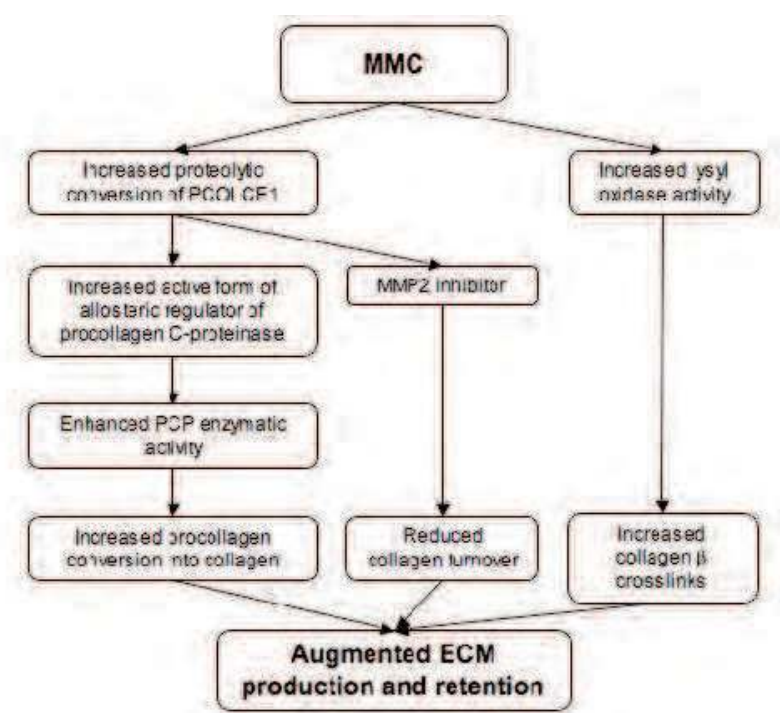

Fig. 8. Mechanisms by which MMC augments extracellular matrix (ECM) production and retention

Other macromolecules that were tried include $100 \mu \mathrm{g} / \mathrm{ml}$ of $670 \mathrm{kDa}$ neutral dextran (NDx), $50 \mathrm{mg} / \mathrm{ml}$ of Fc $400,50 \mathrm{mg} / \mathrm{ml}$ of Fc 70 and $100 \mu \mathrm{g} / \mathrm{ml}$ of $10 \mathrm{kDa}$ DxS. These additional macromolecules were unable to significantly increase collagen deposition. As explained earlier in section 2.5, several factors determine the total excluded-volume effect. By definition, all crowder macromolecules are hydrophilic and therefore the hydrodynamic size $\left(R_{H}\right)$, a parameter that captures the effects of size and hydration, as is, becomes a critical determinant of the volume of exclusion. In addition, for anionic macromolecules, the surface charge further adds on to the effects of size and hydration. We can illustrate this by a simple comparison of the hydrodynamic radii of charged and neutral macromolecules. Anionic Dextran Sulfate of molecular weight $500 \mathrm{kDa}$ with an $\mathrm{R}_{\mathrm{H}}$ of $46 \mathrm{~nm}$ (Table 1) far exceeds the $\mathrm{R}_{\mathrm{H}}$ of a neutral Dextran $(21 \mathrm{~nm})$ that has a much larger molecular weight at $670 \mathrm{kDa}$. This $2.2-$ fold greater increase in radius translates to a 10 -fold increase in volume per molecule and when calculated to the entire population of macromolecules in solution, this translates into a substantial increase in volume occupancy for the charged crowder. Another similar analogy can be described when we compare PSS $(200 \mathrm{kDa})$ with the neutral Dextran $(670 \mathrm{kDa})$. Although PSS is three times lower in MW than NDx, both have a similar $\mathrm{R}_{\mathrm{H}},(21 \mathrm{~nm})$. This highlights the significance of charge to determining the hydrodynamic radius. These biophysical findings highlighting the role of charge were corroborated by the cell culture results, which demonstrated the inability of NDx and Fc 70 and FC 400 as monocrowders to elicit the same degree of collagen deposition when compared with the charged crowders in similar conditions.

Combination of Fc 70 and 400 at $37.5 \mathrm{mg} / \mathrm{ml}$ and $25 \mathrm{mg} / \mathrm{ml}$, respectively, yielded enhanced collagen production due to the synergistic effects of such a mixed crowder cocktail, as described by Harve et. al., 2008. 
When the effects of two Dextran Sulfate species, one at 500kDa and another at $10 \mathrm{kDa}$, were compared, biophysically it was found that the smaller DxS species was undetectable in DLS as the size of this crowder was below the detection threshold. The DxS10 was understandably found to yield minimal amounts of collagen in comparison to its heavier and bigger counterpart. Thus size also does matter when the excluded-volume effects are compared between two charged macromolecules of similar chemical structure.

\begin{tabular}{r|c|c|c|c|c|c} 
& $\begin{array}{c}\text { DxS } \\
500 \mathrm{kDa}\end{array}$ & $\begin{array}{c}\text { PSS } \\
200 \mathrm{kDa}\end{array}$ & $\begin{array}{c}\text { NDx } \\
670 \mathrm{kDa}\end{array}$ & $\begin{array}{c}\text { Fc } \\
400 \mathrm{kDa}\end{array}$ & $\begin{array}{c}\text { Fc } \\
700 \mathrm{kDa}\end{array}$ & $\begin{array}{c}\text { DxS } \\
10 \mathrm{kDa}\end{array}$ \\
\hline Net Charge & Negative & Negative & Neutral & Neutral & Neutral & Negative \\
\hline $\begin{array}{r}\text { Hydro. Radius } \\
(\mathrm{nm})\end{array}$ & 46 & 22 & 21 & 4.5 & 3 & $<1$ \\
\hline
\end{tabular}

Table 1. Hydrodynamic radii of macromolecules 500kDa DxS, PSS, NDx, Fc 400, Fc 70 and 10kDa DxS

\subsection{Why MMC is not equivalent to a simple volume reduction of culture medium}

A question often posed by audiences worldwide is since MMC creates a concentration effect via volume exclusion, why not grow the cells in reduced volumes of culture medium? Would this not lead to a similar concentration of secreted factors like enzymes and reactants?

We designed an experiment to address this question. We grew 50,000 fibroblasts in each well of a 24 well culture dish $(15 \mathrm{~mm}$ diameter) varying the volumes of culture medium from $500 \mu \mathrm{l}$ to $100 \mu \mathrm{l}$. In parallel, we ran one DxS-crowded set of cells in $500 \mu \mathrm{l}$. As to be expected, in the smallest volumes, cell cultures dried out, resulting in cell death. Interestingly, there was an increase of collagen deposition in the $200 \mu \mathrm{l}$ well by 2.2-fold (Fig. 9A). However, this was not substantial compared to the collagen deposition induced in the $500 \mu \mathrm{l}$ well by DxS, which was increased by an order of magnitude in this experiment (Fig. 9A). We calculated the thermodynamic activity of reactants procollagen and its enzymes procollagen C-proteinase in the case of $500 \mu \mathrm{l}$ of DxS-supplemented culture medium, $200 \mu \mathrm{l}$ of uncrowded medium and $100 \mu \mathrm{l}$ of uncrowded medium and found an order of magnitude difference in the $500 \mu \mathrm{l}$ crowded medium in comparison with the latter two cases (Fig. 9B). Such a large difference in the thermodynamic activities could explain the much-enhanced collagen deposition of the crowded culture.

Thus, MMC and the resulting excluding volume effect are far more powerful than simply reducing the volume of medium, and still allow for ample nutrient-rich culture medium in static or bioreactor settings. We should mention here that MMC in our system did not cause appreciable changes of viscosity (Lareu et. al., 2007a) to the culture medium thus eliminating concerns with diffusion issues. 
A $\quad$ B

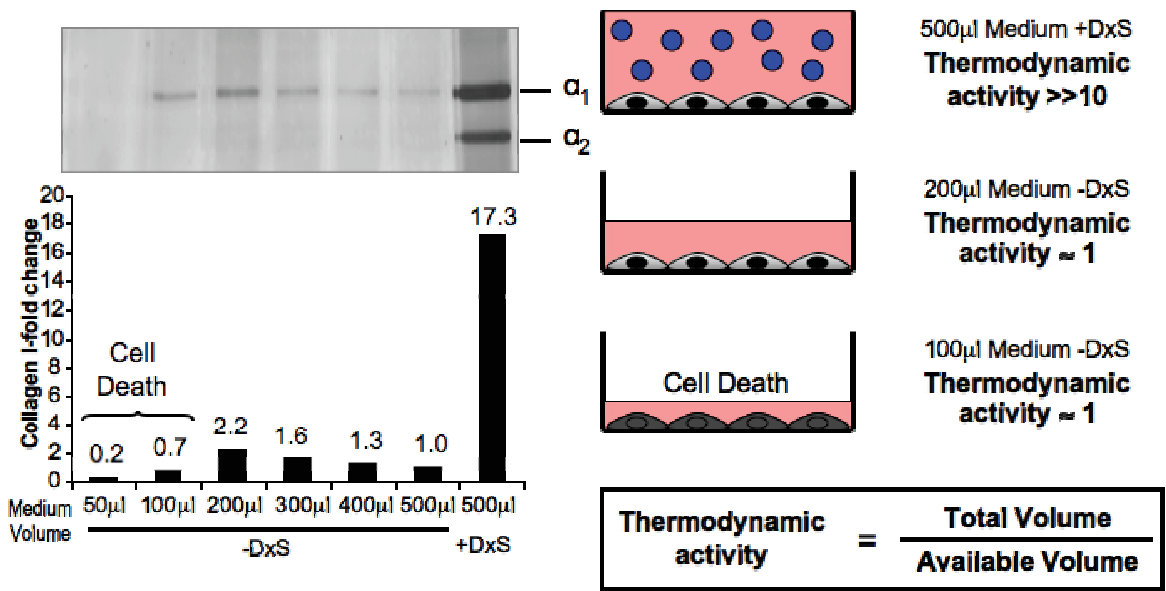

Fig. 9. The reduction of medium volume was unable to enhance collagen deposition to the same degree as the application of MMC. (A) Fibroblast cultures were grown under noncrowded conditions with reduced volumes of medium and showed at best, a 2.2-fold collagen deposition using $200 \mu \mathrm{l}$ of medium. In contrast, DxS-crowded fibroblasts showed a much more enhanced collagen deposition at 17.3-fold. When medium volume was reduced to $100 \mu \mathrm{l}$ and $50 \mu \mathrm{l}$, the wells dried out, resulting in cell death. (B) Thermodynamic activities in $500 \mu \mathrm{l}$ of DxS-crowded medium far exceeds that of $200 \mu \mathrm{l}$ and $100 \mu \mathrm{l}$ of uncrowded fibroblast cultures

\subsection{Increased collagen biosynthesis by MMC for application in connective tissue engineering}

We have shown that MMC can be employed to increase collagen biosynthesis in fibroblast cultures. Because collagen comprises the majority of the extracellular matrix components in vivo, we are able to produce an extracellular matrix in vitro that is more physiological. Our research has brought a technological advancement to the field of connective tissue engineering and other tissue engineering fields as well.

Additionally, we have shown that MMC can be applied in conventional tissue engineering systems comprising scaffolds cultured under static or bioreactor conditions (Lareu et.al. 2007b). We have successfully supplemented DxS into the culture medium of fibroblasts seeded onto Biobrane scaffolds. In comparison to the control, the presence of DxS increased collagen production by $>6$-fold (Fig. 10A.). Biobrane is a biosynthetic wound dressing comprising a silicone film with a nylon fabric partially embedded into the film. Small collagen peptides are coated onto the nylon fabric, which would be broken down during our pepsin-isolation protocol and hence will not contribute to our collagen detection method. The use of Biobrane as a scaffold is a proof-of-concept of the efficacy of employing MMC in connective tissue engineering.

In another culture system, DxS was supplemented into the culture medium of fibroblasts seeded onto poly-L-lactic acid scaffolds under either static conditions in petri dishes, or 
under bioreactor conditions. Under both conditions, DxS was able to increase collagen production by $>6$ fold and 3-fold respectively (Fig. 10B.).
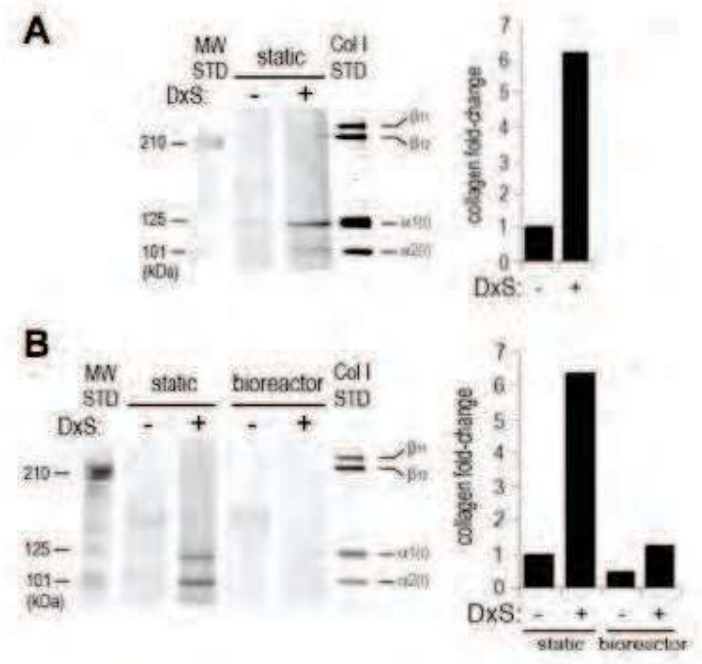

Fig. 10. DxS induced increased collagen production in fibroblast cultures on scaffolds (A) Biobrane and (B) PLLA. PLLA fibroblast cultures were grown under both static and bioreactor conditions. Reprinted from Lareu et. al., 2007b, with permission from the publisher, Elsevier

Thus, we have shown that collagen deposition can be enhanced by the application of MMC in two different scaffold fibroblast culture systems as well as under bioreactor conditions. The application of a biophysical principle, MMC, to solve a tissue engineering problem, is a fine example of how important it is to adopt a multi-disciplinary approach towards solving tissue engineering problems.

The increase in collagen has the potential to benefit most if not all tissue engineering constructs because the protein serves to create cohesion between single cells and cell layers, thereby contributing to mechanical support. Moreover, applying MMC results in an extracellular matrix more similar to that found in vivo, which has the potential to provide biochemical cues such as growth factors, fulfilling a more physiological role in directing the cells.

\subsection{Side observations on crowding - growth behaviour}

We noted significant differences in cell proliferation depending on the crowders used. In the presence of negatively charged DxS, cell proliferation halted, such that there was $12 \%$ less cells in comparison to the uncrowded control in 2 days (unpublished data). In contrast, in the presence of neutral crowders in the Fc cocktail, cell proliferation was increased by $15 \%$ in a time window of 6 days (unpublished data). A possible explanation for this phenomenon could be that these observations do not reflect a true stimulation of proliferation by $\mathrm{Fc}$, but rather a longer proliferation slow-down time as it takes longer under 
these conditions to build up a critical mass of ECM that could move cells into a quiescent state.

\subsection{Current immediate application of MMC in cell culture}

The ability to rapidly create matrix in vivo allowed us to use this technology to build a drug screening tool, the Scar in a Jar (Chen et al, 2009), therefore enabling us to emulate collagen biosynthesis, secretion and deposition in a compressed time window. Thus we can emulate scar formation and test antifibrotic substances in a high content screening setting (Wang et. al., 2009).

\section{Future Challenges}

One of the current challenges faced by tissue engineers is the size and complexity of tissue constructs. We envision that macromolecular crowding can enhance both static and bioreactor settings and induce cells to coat polymeric biomaterials with their own matrix. Organ printing is an emerging field, where pieces of pelleted tissue are printed onto a hydrogel or substrate and fuse together to form a larger construct. Currently, organ printing has enabled the formation of capillary-like tubes from pellets of endothelial cells (Mironov, 2009). The biggest obstacle to this technique is keeping the printed cell constructs stable. These constructs are most likely unstable due to their lack of structural matrix components after printing. Printing into a scaffold is technically challenging, and hence a less preferred option. However, we propose that exposing printed or extruded cell constructs to macromolecular crowders could enhance collagen deposition by these cell pellets themselves, thereby reducing the period of instability and creating a more cohesive tissue construct. A particular way to create coherent cell sheets was pioneered by Teruo Okano and coworkers (Yang et. al., 2007). This technique is based on thermosensitive polymers that switch hydrophilicity according to temperature. This has led to the discovery that cells grown as a hyperconfluent monolayer can be lifted of as a coherent cell sheet by cooling the culture plates. This elegant technique is dependent on a basic ECM containing of fibronectin and collagen $\mathrm{V}$ that lend cohesion to the cell sheet. Unfortunately, it does not appear possible currently to generate matrix-enhanced cell layers and to lift them off the Okano material, as the cells adhere extremely firmly onto the deposited ECM, and the ECM in turn to the polymer. However, we are confident that crowding and cell sheet technology can be combined by designing novel temperature-sensitive cell culture polymers that have a lower critical solution temperature, between $4^{\circ} \mathrm{C}$ and $10^{\circ} \mathrm{C}$ and would be predicted to release a matrix-cell- layer completely. This work is currently work in progress.

\section{Acknowledgments}

We would like to thank Dr Karthik Harve Subramhanya and Clarice Chen, Tissue Modulation Laboratory, for their contributions in this work and critical reading. The authors acknowledge support by the NUS Tissue Engineering Programme and the Bioengineering Programme (Life Science Institute NUS) and the Faculty Research Committee. This work was supported by grants from the National Medical Research Council of Singapore (NMRC grant CPG/003/2004, R-397-000-018-213), the Faculty of Engineering, Office of Research FRC grants (R-397-000-017-112, R-397-000-081-112), Office of Life Science (R-397-000-604- 
101, R-397-000-604-712) Y.P. is a recipient of a graduate research scholarship from the Division of Bioengineering, National University of Singapore.

\section{References}

Bateman, J.F.; Cole, W.G.; Pillow, J.J. \& Ramshaw, J.A.M. (1986). Induction of procollagen processing in fibroblast cultures by neutral polymers. Journal of Biological Chemistry, Vol. 261, No. 9 (Month 1986) 4198-4203, 0021-9258

Chebotareva, N.A.; Kurganov, B.L. \& Livanova, N.B. (2004). Biochemical effects of molecular crowding. Biochemistry (Moscow), Vol. 69, No. 11 (Nov 2004) 1239-1251, 0006-2979

Chen, C.Z.C.; Peng, Y.; Wang, Z.; Fish, P.V.; Kaar, J.L.; Koepsel, R.R.; Russell, A.J.; Lareu, R.R. \& Raghunath, M. (epub, ahead of print) The scar-in-a-jar: studying antifibrotic lead compounds from the epigenetic to extracellular level in a single well. British Journal of Pharmacology, in press, 0007-1188

Ellis, R.J. (2001). Macromolecular crowding: obvious but underappreciated, Trends in Biochemical Sciences, Vol. 26, No. 10 (Month 2001) 597-604, 0968-0004

Harve, K.S.; Vigneshwar, R.; Rajagopalan, R. \& Raghunath, M. (2008) Macromolecular crowding in vitro as means of emulating cellular interiors: when less might be more. Proceedings of the National Academy of Sciences of the United States of America, Vol 105, No. 51 (Dec 2008), E119, 1091-6490

Hojima, Y.; Behta, B.; Romanix, A.M. \& Prockop, D.J. (1994). Cleavage of type I procollagen by C- and N-proteinase is more rapid if the substrate is aggregated with dextran sulfate or polyethylene glycol. Analytical Biochemistry, Vol. 223 (Month 1994), 173$180,0003-2697$

Kielty, C.M \& Grant, M.E., (2002). The collagen family: Structure, assembly, and organization in the extracellular matrix, In: Connective Tissue and its Heritable Disorders Molecular, Genetic, and Medical Aspects, Royce, P.M. \& Steinmann, B. (Ed.), 103-147, Wiley-Liss Inc., 0471221929, New York

Lareu, R.R.; Arsianti, I.; Subramhanya, H.K.; Peng, Y. \& Raghunath, M. (2007a). In vitro Enhancement of Collagen Matrix Formation and Crosslinking for Applications in Tissue Engineering: A Preliminary Study. Tissue Engineering, Vol. 13, No. 2, (February 2007) 385-391, 1937-3341

Lareu, R.R.; Subramhanya, H.K.; Peng, Y.; Benny, P; Chen, C; Wang, Z; Rajagopalan, R \& Raghunath, M. (2007b). Collagen Matrix Deposition is dramatically enhanced In vitro when Crowded with Charged Macromolecules: The Biological Relevance of the Excluded Volume Effect. FEBS Letters, Vol. 581, No. 14 (May 2007) 2709-2714, 0014-5793

Macchiarini, P.; Junebluth, P.; Go, T.; Asnaghi, M.A.; Rees, L.E.; Cogan, T.A.; Dodson, A.; Martorell, J.; Bellini, S.; Parnigotto, P.P.; Dickinson, S.C.; Hollander, A.P.; Mantero, S.; Conconi, M.T. \& Birchall, M.A. (2008). Clinical Transplantation of a TissueEngineered Airway. The Lancet, Vol. 372, No. 9655 (December 2008) 2023-2030, 0140-6736

Minton, A.P. (2001). The influence of macromolecular crowding and macromolecular confinement on biochemical reactions in physiological media. Journal of Biological Chemistry, Vol 276, No. 14 (February 2001) 10577-10580, 0021-9258 
Mironov, V.; Visconti, R.P.; Kasyanov, V.; Forgacs, G.; Drake, C.J. \& Markwald, R.R. (2009). Organ printing: Tissue spheroids as building blocks. Biomaterials Vol. 30, (Jan 2009) 2164-2174, 0142-9612

Mott, J.D.; Thomas, C.L.; Rosenbach, M.T.; Takahara K.; Greenspan, D.S. \& Banda, M.J. (2000). Post-translational proteolystic processing of procollagen C-terminal proteinase enhancer releases a metallo-proteinase inhibitor. Journal of Biological Chemistry, Vol. 275, No. 2 (Jan 2000) 1384-1390, 0021-9258

Murad, S.; Grove, D.; Lindberg, K.A.; Reynolds, G.; Sivarajah, A. \& Pinnell, S.R. (1981). Regulation of collagen synthesis by ascorbic acid. Proceedings of the National Academy of Sciences of the United States of America, Vol. 78, No. 5 (May 1981) 2879$2882,1091-6490$

Sottile, J.; Shi, F.; Rublyevska, I.; Chinag, H.Y.; Lust, J. \& Chandler, J. (2007) Fibronectindependent collagen I deposition modules cell response to fibronectin. American Journal of Physiology Cell Physiology, Vol. 293 (Oct 2007) C1934-C1946, 0363-6143

Wang, Z.; Chen, C.; Finger, S.N.; Kwajah, S.; Jung, M.; Schawz, H.; Swanson, N.; Lareu, R.R. \& Raghunath, M. (2009) Suberoylanilide hydroxamic acid: a potential epigenetic therapeutic agent for lung fibrosis? The European Respiratory Journal, Vol 34, No. 1 (July 2009) 145-155, 1399-3003

Yang, J.; Yamato, M.; Shimizu, T.; Sekine, H.; Ohashi, K.; Kanzaki, M.; Ohki, T.; Nishida, K. \& Okano, T. (2007) Reconstruction of functional tissues with cell sheet engineering. Biomaterials, Vol. 28, No. 34 (Dec 2007) 5033-5043, 0142-9612

Zimmerman, S.B. \& Minton, A.P. (1993) Macromolecular crowding: biochemical, biophysical, and physiological consequences. Annual Review of Biophysics and Biomolecular Structure, Vol. 22 (June 1993), 27-65, 1056-8700 


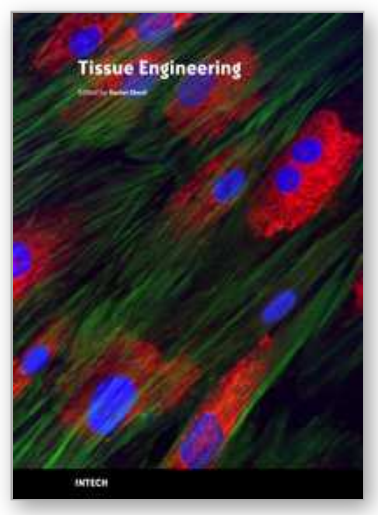

\author{
Tissue Engineering \\ Edited by Daniel Eberli
}

ISBN 978-953-307-079-7

Hard cover, 524 pages

Publisher InTech

Published online 01, March, 2010

Published in print edition March, 2010

The Tissue Engineering approach has major advantages over traditional organ transplantation and circumvents the problem of organ shortage. Tissues that closely match the patient's needs can be reconstructed from readily available biopsies and subsequently be implanted with minimal or no immunogenicity. This eventually conquers several limitations encountered in tissue transplantation approaches. This book serves as a good starting point for anyone interested in the application of Tissue Engineering. It offers a colorful mix of topics, which explain the obstacles and possible solutions for TE applications.

\title{
How to reference
}

In order to correctly reference this scholarly work, feel free to copy and paste the following:

Yanxian Peng and Michael Raghunath (2010). Learning From Nature: Emulating Macromolecular Crowding To Drive Extracellular Matrix Enhancement For The Creation Of Connective Tissue in vitro, Tissue Engineering, Daniel Eberli (Ed.), ISBN: 978-953-307-079-7, InTech, Available from:

http://www.intechopen.com/books/tissue-engineering/learning-from-nature-emulating-macromolecularcrowding-to-drive-extracellular-matrix-enhancement-for

\section{INTECH}

open science | open minds

\section{InTech Europe}

University Campus STeP Ri

Slavka Krautzeka 83/A

51000 Rijeka, Croatia

Phone: +385 (51) 770447

Fax: +385 (51) 686166

www.intechopen.com

\section{InTech China}

Unit 405, Office Block, Hotel Equatorial Shanghai

No.65, Yan An Road (West), Shanghai, 200040, China 中国上海市延安西路65号上海国际贵都大饭店办公楼 405 单元

Phone: +86-21-62489820

Fax: +86-21-62489821 
(C) 2010 The Author(s). Licensee IntechOpen. This chapter is distributed under the terms of the Creative Commons Attribution-NonCommercialShareAlike-3.0 License, which permits use, distribution and reproduction for non-commercial purposes, provided the original is properly cited and derivative works building on this content are distributed under the same license. 